This document is confidential and is proprietary to the American Chemical Society and its authors. Do not copy or disclose without written permission. If you have received this item in error, notify the sender and delete all copies.

\title{
Functional Hybrid Glyconanocapsules by a One-Pot Nanoprecipitation Process
}

\begin{tabular}{|c|c|}
\hline Journal: & Biomacromolecules \\
\hline Manuscript ID & bm-2020-00697c.R2 \\
\hline Manuscript Type: & Article \\
\hline $\begin{array}{r}\text { Date Submitted by the } \\
\text { Author: }\end{array}$ & $n / a$ \\
\hline Complete List of Authors: & $\begin{array}{l}\text { Yan, Xibo; Tianjin University, } \\
\text { Alcouffe, Pierre; Laboratoire des Matériaux Polymères et Biomatériaux, } \\
\text { UMR CNRS 5223 IMP } \\
\text { Bernard, Julien; IMP@INSA, IMP/LMM CNRS, UMR5223 } \\
\text { Ganachaud, Francois; IMP@INSA, IMP UMR 5223 }\end{array}$ \\
\hline
\end{tabular}

\section{SCHOLARONE \\ Manuscripts}




\title{
Functional Hybrid Glyconanocapsules by a One-Pot
}

\section{Nanoprecipitation Process}

\author{
Xibo Yan, ${ }^{*, a}$ Pierre Alcouffe, ${ }^{b}$ Julien Bernard, ${ }^{*, b}$ François Ganachaud*,b \\ a : School of Chemical Engineering and Technology, Tianjin University, Tianjin 300072, China. \\ b: Université de Lyon, Lyon, F-69003, France; INSA-Lyon, IMP, Villeurbanne, F-69621, France; \\ CNRS, UMR 5223, Ingénierie des Matériaux Polymères, Villeurbanne, F-69621, France.
}

\begin{abstract}
Herein, we report on one-pot fabrication of oil-filled nanocapsules wrapped with both RAFT-made glycopolymers and neutral polysaccharides (dextran and pullulan). We have made use of the nanoprecipitation technique, relying on coprecipitation of both oil and polymers in conditions thoroughly established from phase diagrams' interpretation. Mixed mono- or multi-layered nanocapsules were obtained through simultaneous or sequential nanoprecipitation, respectively. Incorporation of synthetic glycopolymer chains allows for precisely tailoring the dimensions of the nanocapsules (size and membrane thickness of the polymeric shell), whereas the insertion of polysaccharides enables to tune the (bio)degradability of the nanocapsules. Shell-functionalized and/or core-loaded capsules could also be achieved in a similar one pot process, by introducing a drug and/or biotin in the organic and aqueous phase, respectively. We finally report magnet-sensitive capsules.
\end{abstract}




\section{INTRODUCTION}

Thanks to their biocompatibility, biodegradability and bioavailability, polysaccharide-based nanoparticles are promising nanomaterials for a large range of applications in pharmaceuticals, cosmetics and food industry. ${ }^{1-3}$ Among these, the preparation of nanometer-scale polysaccharide-based nanocapsules still remains a challenge. ${ }^{3}$ Common routes to this category of glyconanomaterials are (i) covalent linking of hydrophobic (macro)molecular segments to polysaccharides, thus favoring self-assembly of the resulting polymers in water; ${ }^{4-7}$ (ii) combination of oppositely-charged polyelectrolytes with ionic polysaccharides through a Layer-by-Layer (L-b-L) strategy, ${ }^{8-11}$ or (iii) interfacial polymerization/coupling in miniemulsion. ${ }^{12,13}$

Recently, we developed an alternative process to design glyconanocapsules in a straightforward manner. This approach is based on nanoprecipitation via a concomitant deposition and crosslinking of water-soluble polymer chains at the interface of oily liquid templates to spontaneously generate monodisperse, submicron-sized polymeric capsules (Scheme 1A). ${ }^{14}$

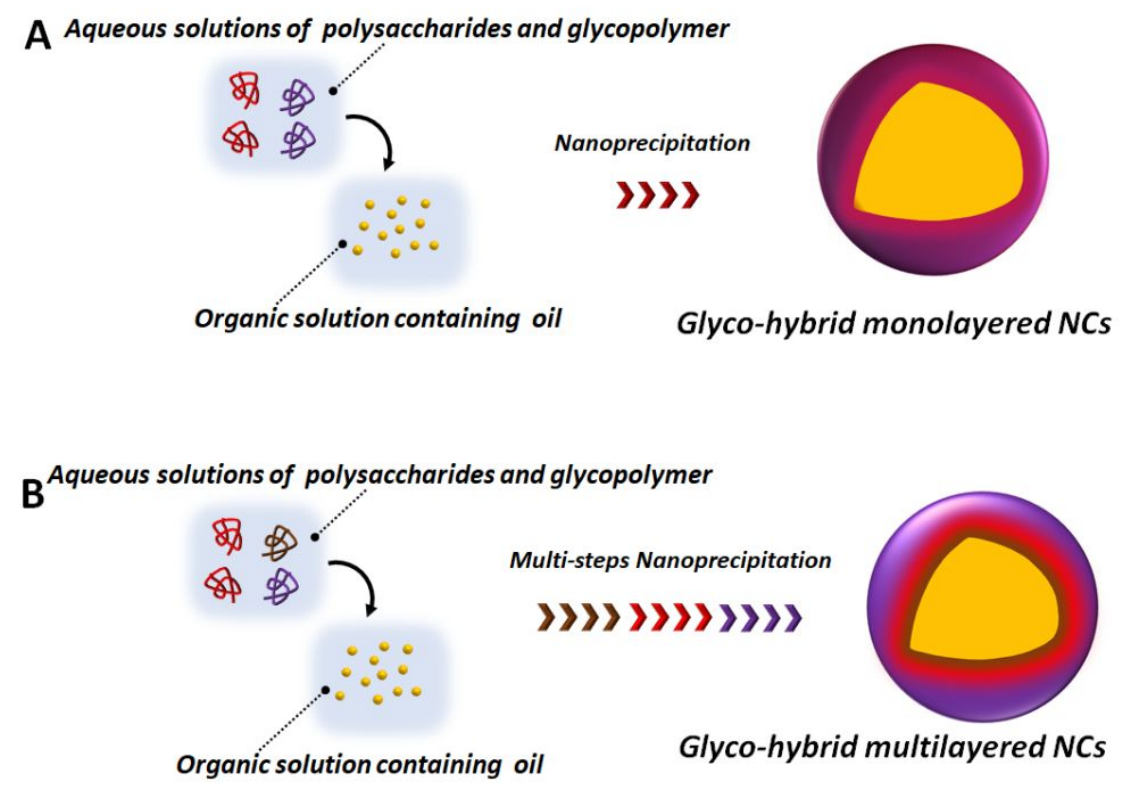

Scheme 1: Schematic illustration of preparation of (A) monolayered polysaccharide hybrid nanocapsules through simultaneous co-nanoprecipitation of the two polymers together with myglyol and (B) multilayered hybrid nanocapsules through sequential nanoprecipitation steps. 
To do so, the establishment of solute/solvent/non-solvent ternary phase diagrams is necessary. Different limits must be pointed out, those delimitating the Ouzo domain (for oil) and the cloud point boundary (for the polymer). The former is a tiny region where oil spontaneously forms droplets, in between the binodal curve and the Ouzo limit (see Figure 1, blue and purple lines, respectively). The latter is the limit above which the hydrophilic polymer is not soluble anymore when adding increasing content of acetone and thus precipitates (Figure 1, grey curves). The zones of co-precipitation that leads to nanocapsules are indicated on Figure 1.

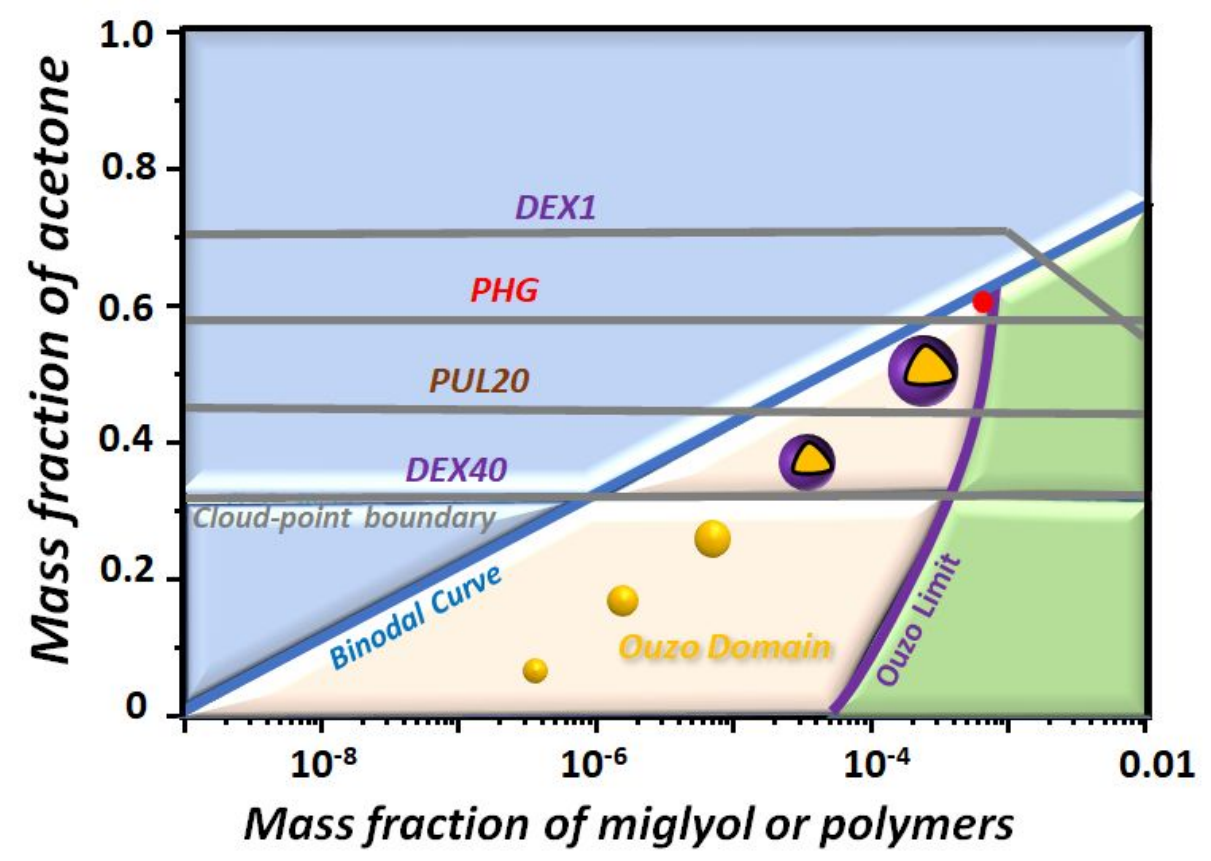

Figure 1. Overlapped phase diagrams of polymer/miglyol/acetone/water mixtures as previously determined in refs [18] and [19]. The cloud-point boundaries in this diagram established with the different polymers are drawn in grey, the Ouzo domain of miglyol in light pink. symbolizes miglyol droplets, $\mathbf{O}$ symbolizes monocomponent polymeric nanocapsules, the red dot indicates the nanoprecipitation conditions chosen here for preparing monocomponent polymeric nanocapsules ( $59 \mathrm{wt} \%$ of acetone, $0.05 \mathrm{wt} \%$ of miglyol and $0.05 \mathrm{wt} \%$ of polymer). Reprinted with permission from ref [18]. Copyright [2015] [Royal Society of Chemistry] and ref [19]. Copyright [2019] [American Chemical Society]. 
We have successfully applied this technique to a range of synthetic polymers, i.e. poly(N-(2hydroxypropyl)methacrylamide), ${ }^{15}$ poly(vinyl alcohol) ${ }^{16}$ or glycopolymers, ${ }^{14,}$ 15, 17,18 and polysaccharides. ${ }^{19}$ We found out that vinyl-based glycopolymers contribute to the rapid stabilization of the oil droplets and eventually produce nanocapsules of moderate size (typically below $150 \mathrm{~nm}$ ) with thin but dense shells. On the other hand, when using polysaccharides to design glyconanocapsules, adsorption of the polymer chains on the oil droplets is probably less favorable, whereas the swelling ratio of the resulting cross-linked polymer membrane tends to be higher (for a given cross-linking density). Consequently, after acetone removal, capsules prepared in the Ouzo domain are typically larger in size (around $200 \mathrm{~nm}$ ). A $100 \mathrm{~nm}$-diameter range can however be attained by constructing diverse hierarchical nanocapsules from neutral and ionic polysaccharides. Such a drop in size is favored by both preventing extended nuclei aggregation during the nanoprecipitation process and increasing the cross-linking density. ${ }^{19}$

In the present study, we propose to prepare hybrid nanocapsules by combining synthetic glycopolymers and commercially available polysaccharides as shell-building blocks. After the solvent shifting conditions have been set up, concomitant or selective nanoprecipitations of these structurally and physical-chemically distinct polymers are performed to design a library of hybrid multicomponent single- or multiple- layered nanocapsules. The idea here is to modulate the balance between glycopolymer and polysaccharide chains content engaged in the nanoprecipitation process in view of tuning the size of the capsules, the thickness of their shell and their functionality, while maintaining their overall biodegradability and permeability. 


\section{EXPERIMENTAL}

\section{Materials}

The main structures of the polymers used here are presented in Table 1. Dextran FP40 $\left(\mathrm{M}_{\mathrm{w}} \sim 40 \mathrm{~kg} / \mathrm{mol}\right.$, $Đ=1.34)$ was purchased from SERVA and Pullulan $20\left(\mathrm{M}_{\mathrm{w}} \sim 20 \mathrm{~kg} / \mathrm{mol}, \mathrm{D}=1.09\right)$ was purchased from Shodex. All other polysaccharides were purchased from Sigma-Aldrich, i.e. Dextran $1\left(\mathrm{M}_{\mathrm{w}} \sim 1 \mathrm{~kg} / \mathrm{mol}\right.$, $\mathrm{D}=1.26$ ), sold as a standard for SEC purposes, Fluorescein isothiocyanate-dextran and Biotin-dextran (both $\mathrm{M}_{\mathrm{w}}$ estimated at $\left.70 \mathrm{~kg} / \mathrm{mol}\right)$. Poly(N-[7-( $\alpha$-D-mannopyranosyloxy)heptyl]meth-acrylamide-co-glycidyl

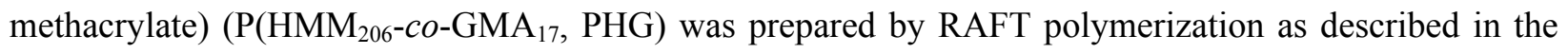
literature $\left(\mathrm{M}_{\mathrm{w}} \sim 77 \mathrm{~kg} / \mathrm{mol}, \mathrm{Đ}=1.10\right) .{ }^{18,20}$

Table 1: Characteristics of polymers used in this study.

\begin{tabular}{|c|c|c|c|c|}
\hline Polymer & Structure & & $\mathrm{Mw}(\mathrm{kg} / \mathrm{mol})$ & 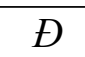 \\
\hline \multirow[t]{4}{*}{ Dextran } & & DEX40 & 40 & 1.34 \\
\hline & $\mathrm{n}=6(\mathrm{DEX} 1), 247(\mathrm{DEX} 40)$ & DEX1 & 1 & 1.26 \\
\hline & & FITC-DEX & 70 & - \\
\hline & & Biotin-DEX & 70 & - \\
\hline \multirow[t]{2}{*}{ Pullulan } & & PUL20 & 20 & 1.09 \\
\hline & $\mathrm{n}=42$ & & & \\
\hline \multirow[t]{2}{*}{$\begin{array}{c}\mathrm{P}\left(\mathrm{HMM}_{206}-\mathrm{co}-\right. \\
\left.\mathrm{GMA}_{17}\right)\end{array}$} & & PHG & 77.1 & 1.10 \\
\hline & $x=206, y=17$ & & & \\
\hline
\end{tabular}


Acetone (99.5\%) was purchased from Carlo Erba and Miglyol 812 from SASOL (Germany). Avidin extracted from egg white (98\%), (S)-(+)-Camptothecin (CPT, 90\%, HPLC), isophorone diisocyanate (IPDI, 98\%), water (HPLC grade) were all purchased from Sigma-Aldrich and used without further purification. Amino-functionalized biotin was obtained by deprotonating biotin ethylenediamine hydrobromide (Sigma-Aldrich, 95\%) by adding 1.5 eq. of triethylamine (SigmaAldrich, 99\%). Turbo beads PEG amine was purchased from TurboBeads (30 mg/mL, $d=30 \mathrm{~nm})$.

Dialysis membranes (Mw cut-off $1000 \mathrm{Da}$ and $300 \mathrm{KDa}$ ) were purchased from Spectrum Laboratories, Inc.

\section{Methods}

Nuclear magnetic resonance (NMR) spectra were recorded on a Bruker Avance III spectrometer (400 MHz) in $\mathrm{D}_{2} \mathrm{O}$ solution at $300 \mathrm{~K}$ and referenced to residual solvent peaks. To characterize the composition of the polymeric shell, trimethyl silyl 3-propionic acid $\mathrm{D}_{4}$ was introduced in $\mathrm{D}_{2} \mathrm{O}$ as an internal reference. The polymer composition in dextran/PHG mixed NCs was calculated by integrating the anomeric protons of glucose unit of dextran $(5.04 \mathrm{ppm})$ and mannose unit of PHG (4.92 ppm).

Particle size measurements were carried out by retroscattering dynamic light scattering (DLS) on a Malvern Instruments Zetasizer nano series instrument using the cumulant method. Aqueous dispersions of glyconanocapsules were prepared at $1 \mathrm{mg} / \mathrm{mL}$, and at least five measurements were made for each sample. Equilibration times of 10 minutes were used before each measurement.

TEM images were recorded on a Philips CM120 electronic microscope, by observations in transmission at an accelerating voltage of $80 \mathrm{kV}$. Samples were prepared by placing a drop of dispersion $(1 \mathrm{mg} / \mathrm{mL})$ onto a Formvar-coated copper grid. The excess solution was carefully blotted off using filter paper and samples were dried for a few minutes before analysis. TEM image 
analysis was performed using ImageJ to basically compare shell thicknesses but not the capsule size distribution. Herein, the analysis of about 50 capsules does not statistically describe the true population dispersal as well as DLS values (see e.g. Figure S1). Also, we suspect that capsules flatten on the grid when drying, thus artificially increasing the distribution size and polydispersity.

Fluorescence spectroscopy analyses were performed on aqueous solutions using a JASCO FP8000 series spectrofluorometer at room temperature.

\section{Cloud point boundaries}

This equilibrium limit corresponds to the solubility limit of the polymer in acetone-water mixtures. ${ }^{14,19}$ It is determined by titrating an aqueous solution of polymer at a precise starting composition with acetone until the mixture turns visually milky (because of the generation of swollen micelles). The cloud point line is drawn from the mass of acetone added at the onset of turbidity for different initial polymer concentrations (see Figure 1, grey curves). The cloud point boundaries of polymers are (acetone mass fractions, wt \%): 58 (PHG), 32 (DEX40), 70 (DEX1), 40 (FITC-DEX and biotin-DEX) and 45 (PUL20), respectively. Note that in the case of polysaccharide samples displaying broad dispersity, solvent shifting must be carried out at higher acetone fractions in order to ensure precipitation of all polymer chains

\section{Preparation of miglyol-filled single layer nanocapsules}

Monocomponent nanocapsules. The preparation of DEX40-based monolayered nanocapsules is given as an example here. DEX $40(0.5 \mathrm{mg})$ was dissolved in $410 \mathrm{mg}$ of water. In a second vial, $0.5 \mathrm{mg}$ of miglyol and $0.05 \mathrm{mg}$ of IPDI $\left(510^{-5}\right)$ were added to $590 \mathrm{mg}$ of acetone. The polymer aqueous solution was then poured into the acetone solution. To avoid substantial swelling of the polymer shell, an additional crosslinking step was undertaken by post-adding $0.05 \mathrm{mg}$ of IPDI in 
$240 \mathrm{mg}$ of acetone. The solutions were left overnight and further characterized by DLS and TEM. The same procedure was used for preparing PHG capsules.

Bicomponent hybrid NCs. The preparation of DEX40/PHG monolayered nanocapsules is taken as an example. $0.5 \mathrm{mg}$ DEX40 and PHG $(0.1 \mathrm{mg}, 0.25 \mathrm{mg}$ or $0.5 \mathrm{mg})$ were dissolved in $410 \mathrm{mg}$ aqueous solution, $0.5 \mathrm{mg}$ miglyol was dissolved in $590 \mathrm{mg}$ acetone with IPDI (24 eq. per overall polymers' chain). The polymer aqueous solution was poured into the acetone solution and the medium turned milky immediately. An additional crosslinking step was undertaken by post-adding $0.05 \mathrm{mg}$ of IPDI in $240 \mathrm{mg}$ of acetone. The solutions were left overnight and then characterized by DLS and TEM. The same procedure was used for PUL20/PHG bicomponent, DEX40/PUL20/PHG and Biotin-DEX/FITC-DEX/PHG tricomponent monolayered capsules.

\section{Preparation of miglyol-filled multilayered hybrid nanocapsules}

DEX40/PHG bilayered NCs. First, $0.5 \mathrm{mg}$ of DEX40 and $0.5 \mathrm{mg}$ of PHG were dissolved in 410 $\mathrm{mg}$ water. Then $0.5 \mathrm{mg}$ miglyol and 24 eq. IPDI dissolved in $590 \mathrm{mg}$ of acetone were added at once in the aqueous phase to selectively precipitate and crosslink DEX40. After 2h, IPDI (24 eq. per polymer chain) in $430 \mathrm{mg}$ acetone were added to increase the ratio of acetone to 0.65 and selectively precipitate PHG. The sample was kept overnight at room temperature. The same procedure was used for preparing mixed PUL20/PHG bilayered capsules.

Biotin-functionalized PHG/DEX1 bilayered NCs. Functionalized NCs were prepared by first dissolving $0.1 \mathrm{mg}$ of DEX1 and $0.5 \mathrm{mg}$ of PHG in $410 \mathrm{mg}$ water. Then $0.5 \mathrm{mg}$ miglyol and $24 \mathrm{eq}$. IPDI were dissolved in $590 \mathrm{mg}$ of acetone and added to precipitate and crosslink PHG. After $2 \mathrm{~h}$, $0.01 \mathrm{mg}$ of amino-functionalized biotin was added into solution and reacted at $40{ }^{\circ} \mathrm{C}$ overnight. After cooling down to r.t., IPDI (24 eq. per polymer chain) in $1000 \mathrm{mg}$ acetone were added to 
increase the ratio of acetone to 0.8 and precipitate DEX1. The sample was kept overnight at room temperature.

PUL20/PHG/DEX1 trilayered NCs. PUL20 (0.5 mg), PHG (0.5 mg), DEX1 (0.5 mg) were first dissolved in $550 \mathrm{mg}$ of water. In a second vial, $0.4 \mathrm{mg}$ of miglyol and $0.05 \mathrm{mg}$ of IPDI were completed with $450 \mathrm{mg}$ of acetone. The polymer aqueous solution was then poured into the acetone solution all at once to precipitate PUL20. After 2 hours, $0.05 \mathrm{mg}$ of IPDI in $572 \mathrm{mg}$ of acetone was added to perform a solvent shift to $65 \%$ of acetone in the solution and precipitate/crosslink PHG. After 2 hours, $0.05 \mathrm{mg}$ of IPDI in $1200 \mathrm{mg}$ of acetone was added to perform another solvent shift to $80 \%$ of acetone in the solution and precipitate/crosslink DEX1. The solutions were left overnight and further characterized by DLS and TEM.

\section{Other procedures}

Preparation of magnetic NCs. After formation of FITC-DEX/Biotin-DEX/PHG mixed nanocapsules, $0.02 \mathrm{~mL}$ of amino-turbobead $(30 \mathrm{mg} / \mathrm{mL}, d=30 \mathrm{~nm})$ was added into the solution and reacted overnight at $40^{\circ} \mathrm{C}$. The magnetic NP-grafted nanocapsules were characterized by DLS and TEM. Purification of the nanocapsules was performed through magnetic separation (x 3 aqueous solution).

Encapsulation of camptothecin (CPT). The preparation of biotin-DEX/FITC-DEX/PHG mixed NCs is the same as above except that $0.01 \mathrm{wt} \% \mathrm{CPT}$ was added in acetone. To calculate the encapsulation efficiency, the nanocapsules were taken back on $100 \mathrm{~nm}$-cut centrifugation filters. After evaporation and freeze drying, the mixture was redissolved in pure acetone $(2 \mathrm{~mL})$. The concentration of CPT in solution was determined thanks to a calibration curve of CPT in acetone.

$$
\text { Encapsulation Efficiency }(\%)=\frac{\text { Total CPT-Free CPT }}{\text { Total } C P T} \times 100 \%
$$


Enzymatic degradation. After formation of FITC-DEX/Biotin-DEX/PHG mixed nanocapsules, acetone was evaporated and then $1 \mathrm{U}$ dextranase was added into the aqueous solution. The temperature was raised to $37^{\circ} \mathrm{C}$ and the degradation studied overnight.

Streptavidin-triggered aggregation experiments. After formation of biotin-functionalized nanocapsules, the solution $(1 \mathrm{~mL})$ was purified by dialysis for 3 days. Then, $0.01 \mathrm{mg}$ of avidin in $0.1 \mathrm{~mL}$ of water was added into the glyconanocapsule solution and the evolution of the aggregated complex was analyzed by DLS.

\section{RESULTS AND DISCUSSION}

\section{Phase diagrams}

In the present study, we made use of three water-soluble neutral polymers whose structures and features are given in Table 1: one synthetic functional glycopolymer, poly(N-[7-( $\alpha-\mathrm{D}-$ mannopyranosyloxy)heptyl]methacryl-amide-co-glycidyl methacrylate) $\left(\mathrm{P}\left(\mathrm{HMM}_{206}-\mathrm{co}\right.\right.$-GMA $\left.\mathrm{GM}_{17}\right)$, PHG) whose synthesis and use was reported in a previous study, ${ }^{18}$ and two sorts of polysaccharides, dextran (two weight average molar masses, 1 and $40 \mathrm{~kg} / \mathrm{mol}$ ) and pullulan (20 kg/mol) (Table 1).

Previously established polymer/miglyol 812/acetone/water and polymer/acetone/water phase diagrams are surimposed in Figure 1. ${ }^{18}, 19$ The cloud point boundaries indicate the critical solubility limit of the different polymers, at acetone mass fractions of 58, 45 and $32 \mathrm{wt} \%$ for PHG, PUL20 and DEX40, respectively. Note that, because the molar mass distribution of some polysaccharide samples are relatively broad, solvent shifting should be performed far above this boundary to precipitate both low and large molar mass polymers. Here all boundaries (but DEX1's) overlap with the Ouzo region identified for the miglyol/acetone/water ternary system where nanocapsules are straightforwardly formed via nanoprecipitation. 
As references for the following results, monocomponent NCs of DEX40, PHG and PUL20 were first produced, (see supporting information, Figure S1). Under such conditions, co-precipitation of miglyol and PHG generated nanocapsules of at least half size compared to miglyol/polysaccharide couples (about 120 against 220 and $386 \mathrm{~nm}$ for PHG, DEX and PUL, respectively).

\section{Hybrid glycopolymer/polysaccharide oil-filled monolayered capsules}

Nanoprecipitations involving a combination of two polymers were then performed first with a weight ratio of PHG to dextran of 50/50 (see principle in Scheme 1). The conditions of solvent shifting in the Ouzo region where both polymers and oil precipitate were determined from the phase diagrams, i.e. $59 \mathrm{wt} \%$ of acetone, $0.05 \mathrm{wt} \%$ of miglyol and $0.1 \mathrm{wt} \%$ of polymers in final composition (see red dot in Figure 1). To do so, an aqueous solution of the two polymers was rapidly poured into acetone solution containing miglyol and $0.01 \mathrm{wt} \%$ of isophorone diisocyanate as cross-linker (IPDI). IPDI has been chosen for its very low reactivity towards water but efficient reaction with alcohol groups..$^{21}$

After microphase separation, the blend of polymers was concomitantly cross-linked at the miglyol/water interface through a rapid isocyanate/alcohol reaction. A second cross-linking step was operated $(0.01 \mathrm{wt} \%$ IPDI in solution in $240 \mathrm{mg}$ of acetone introduced $120 \mathrm{~min}$ after nanoprecipitation) to avoid extensive shell swelling after removal of acetone. ${ }^{19}$ TEM pictures confirmed the formation of core/shell nanostructures comprised of liquid miglyol core and solid mixed polymer shell (Figure 2A). Nanocapsules features closely reproduce those of the PHG monocomponent NCs, with DLS' $z$-average diameter of $139 \mathrm{~nm}$ and DLS's narrow size distribution $(\mathrm{PDI}=0.04)$. TEM photos show a larger variety of capsule sizes than DLS indicates: 
we infer that it comes from the flattening of the capsules on the TEM grid, according to their variable rigidity, and the degree of shrinking of the hydrophilic polymer shell, both of which vary from one polymer to another (see also Figure S1). ${ }^{1} \mathrm{H}$ NMR analysis of the resulting nanocapsules in $\mathrm{D}_{2} \mathrm{O}$ (characteristic peaks at 4.92 and $5.04 \mathrm{ppm}$ corresponding to the anomeric proton of mannose in PHG and glucose unit in dextran, see Figure S2) clearly confirmed the mixed composition of the polymer membrane, in agreement with initial polymer compositions in water phase.
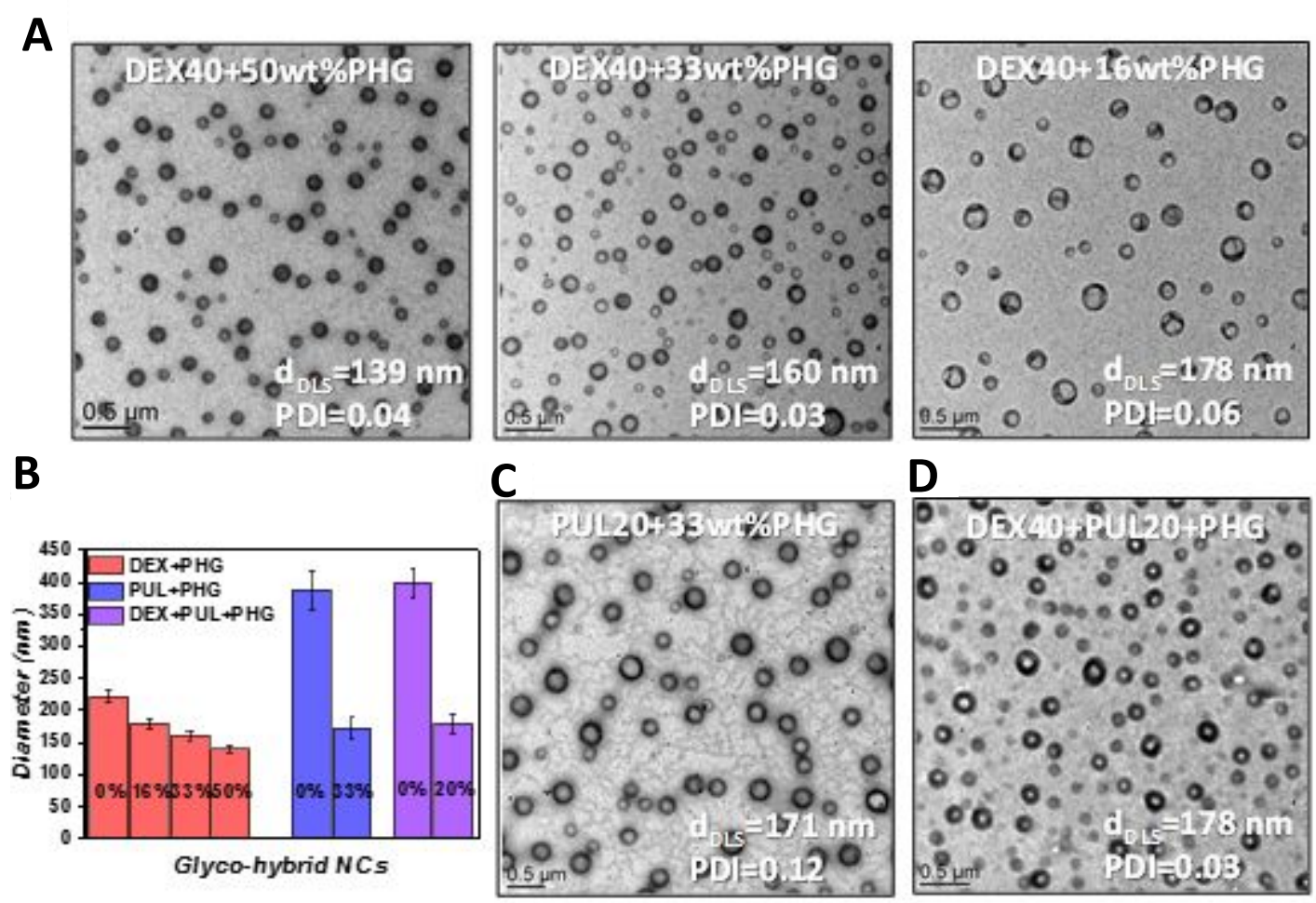

Figure 2. A. TEM images and DLS data of DEX40-PHG (50/50, 84/16, 67/33 wt \%) hybrid nanocapsules; B. Diameter evolution (DLS) of hybrid PHG/polysaccharide nanocapsules as a function of composition in DEX40-PHG, PUL20-PHG bicomponent and DEX40-PUL20-PHG tricomponent systems. Values inside each bar represent the weight ratio of PHG in the polymer mixture; TEM images of corresponding PUL20-PHG (67/33 wt \%) (C) and DEX40-PUL20-PHG (40/40/20 wt \%) (D) hybrid nanocapsules. 
Incorporation of increasing synthetic glycopolymer content resulted in a significant decrease of the size compared to monocomponent dextran-based nanocapsules (weight ratio of dextran/glycopolymer from 84/16, 67/33 to 50/50, Figure 2A). We also measured the thickness of the shells by TEM and did not observe significant variations of dimensions among the bicomponent samples (average thickness of $40 \mathrm{~nm}$, against 43 and $32 \mathrm{~nm}$ for monocomponent NCs of dextran and PHG, respectively).

As emulsification occurs spontaneously after the solvent shifting, we monitored the evolution of dimensions of the nano-objects being formed by DLS (Figure S3). The presence of glycopolymer chains ensures an excellent stabilization of the NCs whose size remains mostly constant from the beginning. In contrast, polysaccharide-coated oily particles only stabilized in size after 5-10 mins. This contrasting behavior may be attributed to the marked amphiphilic character of the glycopolymer, whose preferred adsorption at the $\mathrm{O} / \mathrm{W}$ interface would generate a compact polymer layer (hardly swollen) physically limiting miglyol diffusion by Ostwald ripening. ${ }^{22-24}$ In the literature, it was already shown that dextran hardly decrease the surface tension of water (less than $1 \mathrm{~mJ} / \mathrm{m}^{2}$ for concentrated solution of dextran of $\left.50 \mathrm{~g} / \mathrm{L}\right)^{25}$ whereas glucose functionalized polymers act as efficient dispersants (for instance, glycopolysiloxane solutions decrease the surface tension of water down to $\left.35 \mathrm{~mJ} / \mathrm{m}^{2}\right)^{26}$. Moreover, we cannot exclude that crosslinking by IPDI would be more effective on the glycopolymer than on polysaccharide, thus creating a chemical barrier against miglyol release.

Accordingly, preparing pullulan-PHG mixed nanocapsules allowed reducing NC size from 386 (pullulan-based NCs) to $171 \mathrm{~nm}$ (mixed NCs, weight ratio of pullulan/PHG=67/33, Figures 2B and 2C). Similarly, simultaneous nanoprecipitation of all three polymers in the Ouzo region, i.e. $59 \mathrm{wt} \%$ of acetone, $0.05 \mathrm{wt} \%$ of miglyol, and $0.125 \mathrm{wt} \%$ of polymers with a weight ratio of 
dextran/pullulan/glycopolymer of 40/40/20 generated hybrid NCs with a diameter of $178 \mathrm{~nm}$ $(\mathrm{PDI}=0.03)$ (Figures 2B and 2D).

\section{Functional monolayered hybrid glyconanocapsules}

Next, we precipitated biotin-dextran and FITC-dextran of higher molar mass $(70 \mathrm{~kg} / \mathrm{mol})$ together with PHG (Figure 3). The resulting nanocapsules of $166 \mathrm{~nm}$ in diameter (PDI=0.02) exhibited typical FITC fluorescence emission at $521 \mathrm{~nm}$ (Figure 3B) and strong interaction with tetrameric avidin (as assessed by the formation of large aggregates of nanocapsules, Figure 3C).

In this experiment, a hydrophobic anticancer drug, camptothecin (CPT), was entrapped in the miglyol core by adding it into the organic phase prior to nanoprecipitation (Figure S4). Thanks to the presence of polysaccharide chains homogeneously distributed within mixed polymer shell (67 wt \% content), the hybrid nanocapsules were rapidly destroyed through biodegradation of the polysaccharide triggered by dextranase (Figure 3D); these enzyme-responsive NCs thus release the loaded drug in an externally-triggered manner.

Besides, the epoxide groups distributed along the glycopolymer chains can be used as handles for post-functionalization of the shell. Polymer-inorganic magnetic NPs hybrid nanocapsules (Figure 3E) with a diameter of $234 \mathrm{~nm}$ were further prepared through post-modification with amino-functionalized carbon-coated cobalt magnetic nanoparticles $\left(d=30 \mathrm{~nm}\right.$, at $40^{\circ} \mathrm{C}$ in water).

\section{Multilayered hybrid glyconanocapsules}

Taking advantage of dissimilar cloud point boundaries of polysaccharide and glycopolymer chains, we further programmed the synthesis of multilayered nanocapsules by sequential precipitations of several populations of polymers originally dissolved in the aqueous solution (Scheme 1). No purification was required after successive additions of acetone, owing to selective and quantitative precipitation of each population of polymer chains. 
A

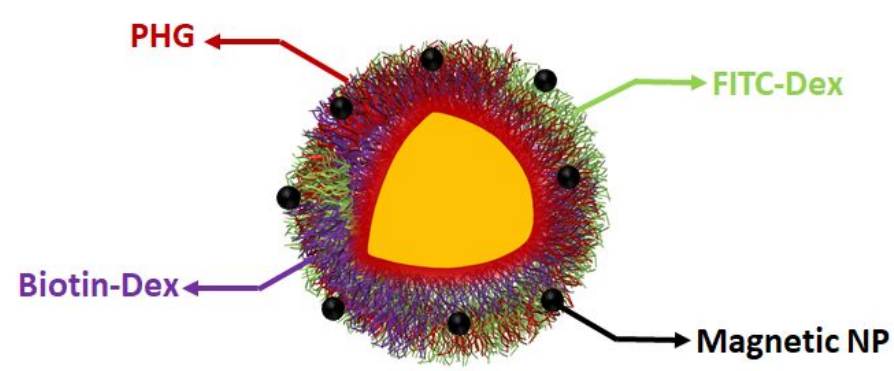

B

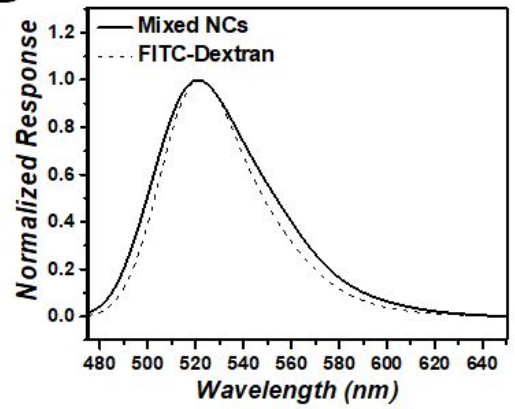

D

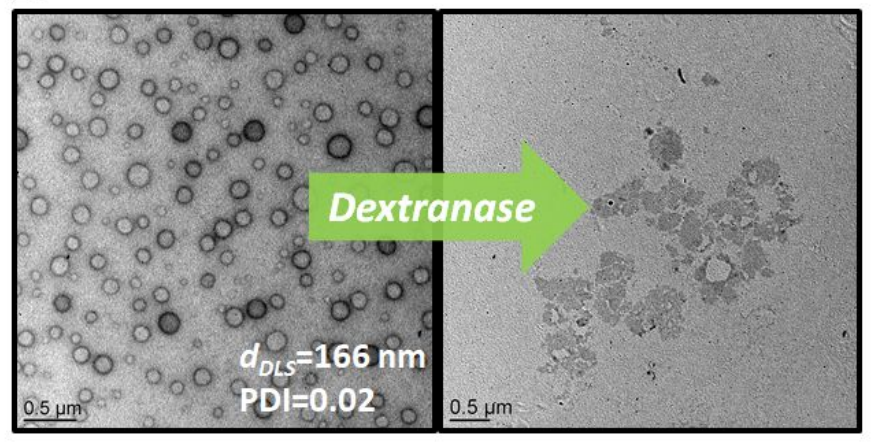

C
Magnetic Dextran Hybrid Nanocapsules

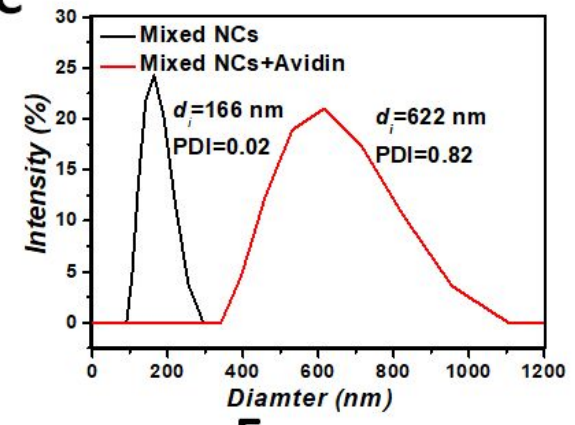

E

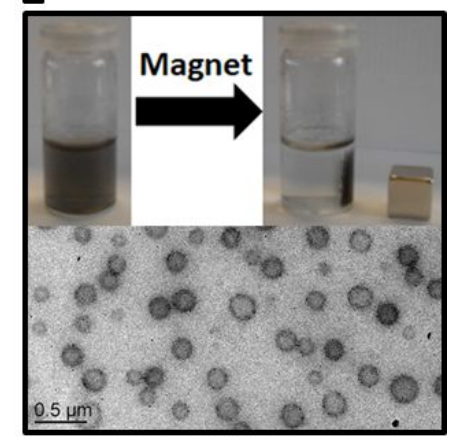

Figure 3. A. Scheme of magnetic responsive FITC-Dex/biotin-Dex/PHG mixed NCs; B. Fluorescence emission spectra of an aqueous solution of free FITC-Dextran and of FITCDex/biotin-Dex/PHG mixed NCs; C. Aggregation of FITC-Dex/biotin-Dex/PHG mixed NCs in the presence of avidin, as observed by DLS; D. Morphology evolution of Biotin-DEX/FITCDEX/PHG mixed NCs after exposure to dextranase; E. TEM picture of magnetic responsive FITCDex/biotin-Dex/PHG mixed NCs (bottom) and their magnetically-driven separation from the aqueous solution (top).

The DEX40/PHG bilayered system is first presented as an example. The two polymers were initially introduced in the water phase. Operating solvent shifting at $50 \mathrm{wt} \%$ of acetone, i.e. below 
and above the cloud point boundary of PHG and DEX40, respectively, selectively precipitated miglyol and dextran into nanocapsules of $164 \mathrm{~nm}$ in diameter under such conditions. The low diameters observed here suggest that a fraction of the amphiphilic glycopolymer chains adsorbs on dextran/water interfaces. Still, the large majority of PHG chains remain in water, as seen by the dark dots on the TEM photograph attributed to the free PHG chains coming out as dense nanoparticles when drying the NC dispersion on the TEM grid (Figure 4A). A second volume of acetone (to finally reach $65 \mathrm{wt} \%)$ with IPDI $(0.05 \mathrm{wt} \%)$ was then poured into the dispersion to trigger the deposition of the free PHG chains on the top of the dextran layer and its cross-linking, resulting in a clean dispersion of bilayered nanocapsules $\left(d_{z}=132 \mathrm{~nm}\right)$. The shell thickness increased here from 20 to $30 \mathrm{~nm}$.

To confirm the coating of one layer onto the other, we subsequently constructed biotinfunctionalized PHG/DEX1 bilayered NCs by sequential nanoprecipitation steps (Figure S5 and Figure 4B). Biotin-functionalized PHG monolayered NCs $\left(d_{z \text { DLs }}=219 \mathrm{~nm}\right)$ were generated by solvent shifting at $59 \mathrm{wt} \%$ of acetone and post-functionalization with biotin ethylenediamine (1 eq. per chain, $40^{\circ} \mathrm{C}$ in water). These NCs were able to multivalently interact with avidin to form large aggregates (Figure 4B). Further enrichment of the medium in acetone (to cross the cloud point of DEX1, acetone mass fraction of $80 \mathrm{wt} \%$ and $0.01 \mathrm{wt} \%$ IPDI) resulted in the formation of NCs with $d_{z \text { DLS }}$ around $180 \mathrm{~nm}$. Contrary to their precursors, the resulting NCs were no longer sensitive to the addition of avidin in the dispersion confirming de facto the deposition of a (second) layer of dextran burying the biotin residues within the contracted polymer shell. The general applicability of the approach was confirmed by facile fabrication of bilayered PUL/PHG (Figure S6) and trilayered PUL20/PHG/DEX1 nanocapsules (Figure 4C). 
In all these experiments, the polymer shell progressively thickens after each layer deposition (e.g. from 37 to $60 \mathrm{~nm}$ as measured on TEM photos for trilayer NCs, Figure 4C), thus expectedly decreasing the NC permeability. ${ }^{19}$ On the other hand, the average diameters of the nanocapsules decrease from the first to second layer depositions, as observed by DLS measurements here (see Figures $4 \mathrm{~B}$ and $4 \mathrm{C}$ ) and before. ${ }^{19}$ This is possibly due to a reduced swelling of surrounding polymer chains when increasing the crosslinks density within the shell. This is also relevant with the fact that the diameter of NCs remains the same from the $2^{\text {nd }}$ to $3^{\text {rd }}$ depositions (Figure 4C).

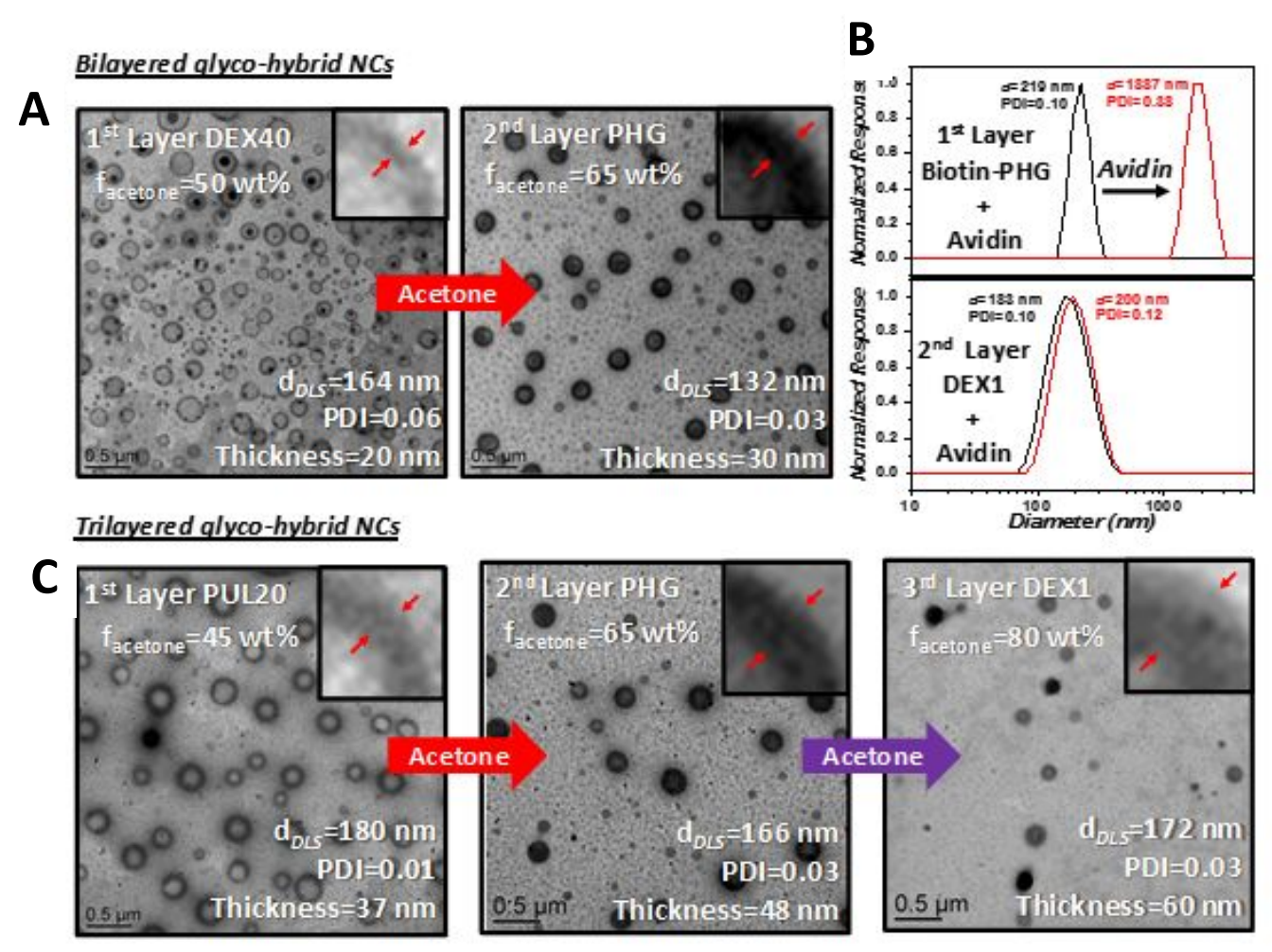

Figure 4. A. TEM photos of first and second building steps of DEX40/PHG bilayered NCs (Inset: zoomed images (10X) of polymeric shell); B. Diameter evolution (DLS) of biotin-functionalized PHG monolayered NCs and biotin functionalized-PHG/DEX1 bilayered NCs in the presence of avidin; C. TEM photos of first, second and third building steps of PUL20/PHG/DEX1 trilayered NCs (Inset: zoomed images (10X) of polymeric shell).

Note finally that the incorporation of glycopolymer chains during the first solvent shifting plays a key role to rapidly stabilize the oil templates and minimize the overall dimensions of the NCs in 
sequential nanoprecipitation process (Figure S7) ${ }^{24}$ As previously reported for AIE probes, ${ }^{27}$ and also illustrated in Figure $\mathbf{S 8}$ with dextran-based NCs, this process can be easily transferred to the gram-scale without modification of NCs' characteristics.

\section{CONCLUSION}

We have reported here a simple and straightforward method for the preparation of hybrid glyconanocapsules. Taking advantage of distinct phase behaviors, synthetic polymer and natural polysaccharides can be controllably precipitated concomitantly and/or selectively to generate mono- or multi-layered glyconanocapsules through simultaneous or sequential nanoprecipitation, respectively. This technique permits convenient prediction and programmed design of polysaccharide-based hybrid nanomaterials with high versatility in terms of size, composition, polymer shell thickness, encapsulation and functionalization. We prepared here nanocapsules of complex architectures with different layers of polymers, loaded with a drug (camptothecin) and covered by biomarker (biotin) or magnetic nanoparticles chemically attached to the polymer chains. Mixing synthetic glycopolymers with natural polysaccharides should allow modulating enzyme-driven biodegradation and cargo release while keeping the overall shape of the capsules, a new feature that was not obtained in our previous published systems. Next study in our laboratory will look at the controlled release of the active cargo using the responsive manner that these nanocapsules endorse. 


\section{ASSOCIATED CONTENT}

\section{Supporting Information.}

The supporting information is available free of charge on the ACS Publications website.

DLS results and TEM images of nanocapsules, ${ }^{1} \mathrm{H}$ NMR of nanocapsules, size evolution of nanocapsules as a function of time, fluorescence spectra of camptothecin-loaded nanocapsules.

\section{AUTHOR INFORMATION}

\section{Corresponding Author}

Email: xiboyan@tju.edu.cn (X. Y.)

julien.bernard@insa-lyon.fr (J. B.)

francois.ganachaud@insa-lyon.fr (F. G.)

\section{Author Contributions}

The manuscript was written through contributions of all authors. All authors have given approval to the final version of the manuscript.

\section{Notes \\ The authors declare no competing financial interest.}

\section{ACKNOWLEDGMENT}

We thank the support from the National Natural Science Foundation of China (Grant 21902117).

JB thanks the French Agency for National Research (ANR) (PREPROPOSAL, ANR-15-CE090021). The authors thank Centre Technologique des Microstructures (CT $\mu)$ for their help with the TEM microscopy. 


\section{REFERENCES}

(1) Lemarchand, C.; Gref, R.; Couvreur, P. Polysaccharide-decorated nanoparticles. Eur. J. Pharm. Biopharm. 2004, 58, 327-341.

(2) Liu, Z.; Jiao, Y.; Wang, Y.; Zhou, C.; Zhang, Z. Polysaccharides-based nanoparticles as drug delivery systems. Adv. Drug Deliv. Rev. 2008, 60, 1650-1662.

(3) Kang, B.; Opatz, T.; Landfester, K.; Wurm, F. R. Carbohydrate nanocarriers in biomedical applications: functionalization and construction. Chem. Soc. Rev. 2015, 44, 8301-8325.

(4) Schatz, C.; Louguet, S.; Le Meins, J.-F.; Lecommandoux, S. Polysaccharide-blockpolypeptide Copolymer Vesicles: Towards Synthetic Viral Capsids. Angew. Chem. Int. Ed. 2009, $48,2572-2575$.

(5) Alibolandi, M.; Alabdollah, F.; Sadeghi, F.; Mohammadi, M.; Abnous, K.; Ramezani, M.; Hadizadeh, F. Dextran-b-poly(lactide-co-glycolide) polymersome for oral delivery of insulin: In vitro and in vivo evaluation. J. Control. Release 2016, 227, 58-70.

(6) Ferji, K.; Venturini, P.; Cleymand, F.; Chassenieux, C.; Six, J.-L. In situ glyco-nanostructure formulation via photo-polymerization induced self-assembly. Polym. Chem. 2018, 9, 2868-2872.

(7) Zhou, C.; Wang, M.; Zou, K.; Chen, J.; Zhu, Y.; Du, J. Antibacterial Polypeptide-Grafted Chitosan-Based Nanocapsules As an "Armed" Carrier of Anticancer and Antiepileptic Drugs. ACS Macro Lett. 2013, 2, 1021-1025.

(8) Shi, D.; Ran, M.; Zhang, L.; Huang, H.; Li, X.; Chen, M.; Akashi, M. Fabrication of Biobased Polyelectrolyte Capsules and Their Application for Glucose-Triggered Insulin Delivery. ACS Appl. Mater. Interfaces 2016, 8, 13688-13697. 
(9) Kwag, D. S.; Oh, K. T.; Lee, E. S. Facile synthesis of multilayered polysaccharidic vesicles. J. Control. Release 2014, 187, 83-90.

(10) Guo, H.; Guo, Q.; Chu, T.; Zhang, X.; Wu, Z.; Yu, D. Glucose-sensitive polyelectrolyte nanocapsules based on layer-by-layer technique for protein drug delivery. J. Mater. Sci. Mater. Med. 2014, 25, 121-129.

(11) Chen, M.-X.; Li, B.-K.; Yin, D.-K.; Liang, J.; Li, S.-S.; Peng, D.-Y. Layer-by-layer assembly of chitosan stabilized multilayered liposomes for paclitaxel delivery. Carbohydr. Polym. 2014, 111, 298-304.

(12) Marie, E.; Landfester, K.; Antonietti, M. Synthesis of Chitosan-Stabilized Polymer Dispersions, Capsules, and Chitosan Grafting Products via Miniemulsion. Biomacromolecules 2002, 3, 475-481.

(13) He, W.; Parowatkin, M.; Mailänder, V.; Flechtner-Mors, M.; Graf, R.; Best, A.; Koynov, K.; Mohr, K.; Ziener, U.; Landfester, K.; Crespy, D. Nanocarrier for Oral Peptide Delivery Produced by Polyelectrolyte Complexation in Nanoconfinement. Biomacromolecules 2015, 16, $2282-2287$.

(14) Yan, X.; Delgado, M.; Fu, A.; Alcouffe, P.; Gouin, S. G.; Fleury, E.; Katz, J. L.; Ganachaud, F.; Bernard, J. Simple but Precise Engineering of Functional Nanocapsules through Nanoprecipitation. Angew. Chem. Int. Ed. 2014, 53, 6910-6913.

(15) Yan, X.; Ramos, R.; Hoibian, E.; Soulage, C.; Alcouffe, P.; Ganachaud, F.; Bernard, J. Nanoprecipitation of PHPMA (Co)Polymers into Nanocapsules Displaying Tunable Compositions, Dimensions, and Surface Properties. ACS Macro Lett. 2017, 6, 447-451. 
(16) Chai, L. Rapid access to functional oil-filled nanocapsules through nanoprecipitation. (Ph.D thesis), 2019, Université de Lyon, France.

(17) Yan, X.; Alcouffe, P.; Sudre, G.; David, L.; Bernard, J.; Ganachaud, F. Modular construction of single-component polymer nanocapsules through a one-step surfactant-free microemulsion templated synthesis. Chem. Comm. 2017, 53, 1401-1404.

(18) Yan, X.; Sivignon, A.; Alcouffe, P.; Burdin, B.; Favre-Bonte, S.; Bilyy, R.; Barnich, N.; Fleury, E.; Ganachaud, F.; Bernard, J. Brilliant glyconanocapsules for trapping of bacteria. Chem. Comm. 2015, 51, 13193-13196.

(19) Yan, X.; Ramos, R. A. N. S.; Alcouffe, P.; Munoz, L. E.; Bilyy, R. O.; Ganachaud, F.; Bernard, J. Programmable Hierarchical Construction of Mixed/Multilayered Polysaccharide Nanocapsules through Simultaneous/Sequential Nanoprecipitation Steps. Biomacromolecules 2019, 20, 3915-3923.

(20) Yan, X.; Sivignon, A.; Barnich, N.; Gouin, S. G.; Bouckaert, J.; Fleury, E.; Bernard, J. A library of heptyl mannose-functionalized copolymers with distinct compositions, microstructures and neighboring non-sugar motifs as potent antiadhesives of type 1 piliated E. coli. Polym. Chem. 2016, 7, 2674-2683.

(21) Torini, L.; Argillier, J. F.; Zydowicz, N. Interfacial Polycondensation Encapsulation in Miniemulsion. Macromolecules 2005, 38, 3225-3236.

(22) Dadyburjor, D. B.; Ruckenstein, E. Kinetics of Ostwald ripening. J. Cryst. Growth 1977, 40, 279-290. 
(23) Liu, Y.; Kathan, K.; Saad, W.; Prud'homme, R. K. Ostwald Ripening of beta-Carotene Nanoparticles. Phys. Rev. Lett. 2007, 98, 036102.

(24) Mora-Huertas, C. E.; Fessi, H.; Elaissari, A. Influence of process and formulation parameters on the formation of submicron particles by solvent displacement and emulsificationdiffusion methods: Critical comparison. Adv. Colloid. Interface. Sci. 2011, 163, 90-122.

(25) Hoorfar, M.; Kurz, M. A.; Policova, Z.; Hair, M. L.; Neumann, A. W. Do Polysaccharides Such as Dextran and Their Monomers Really Increase the Surface Tension of Water? Langmuir 2006, 22, 52-56.

(26) EL-Sukkary, M. M. A.; Ismail, D. A.; El Rayes, S. M.; Saad, M. A. Synthesis and evaluation of polysiloxane derivatives. Egypt. J. Pet. 2014, 23, 361-366.

(27) Yan, X.; Remond, M.; Zheng, Z.; Hoibian, E.; Soulage, C.; Chambert, S.; Andraud, C.; Van der Sanden, B.; Ganachaud, F.; Bretonnière, Y.; Bernard, J. General and Scalable Approach to Bright, Stable, and Functional AIE Fluorogen Colloidal Nanocrystals for in Vivo Imaging. ACS Appl. Mater. Interfaces 2018, 10, 25154-25165. 


\section{TOC graph}

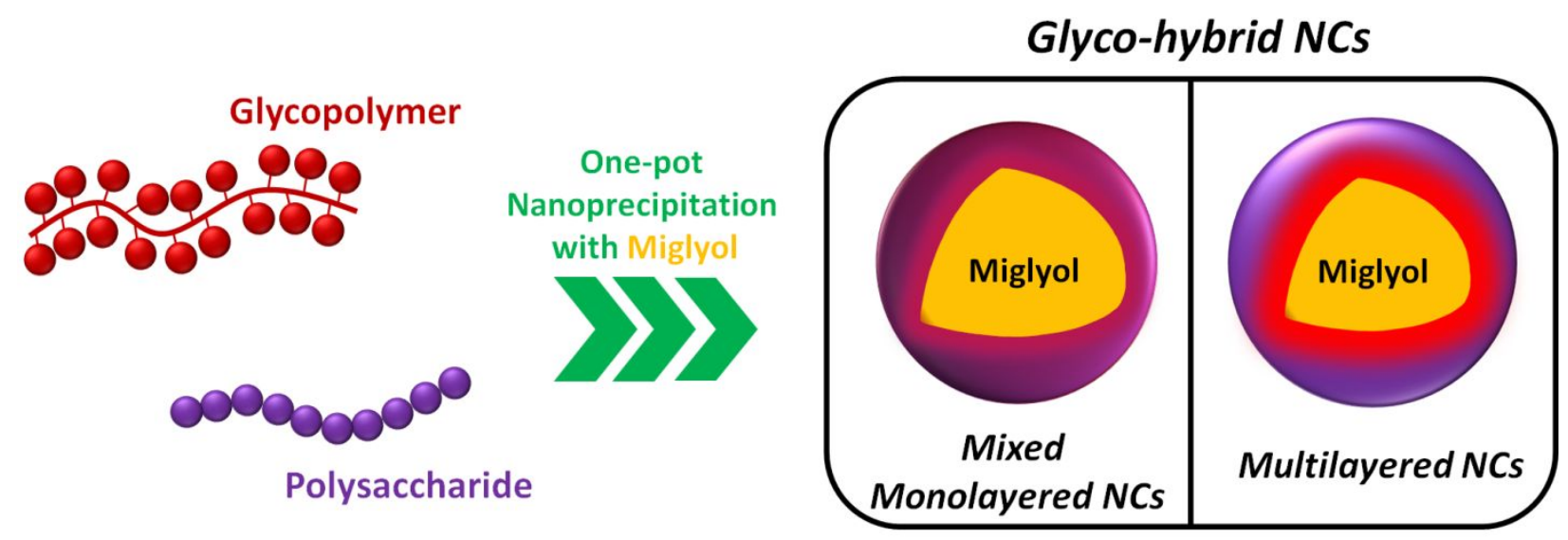

